\title{
Interdisciplinariedad y convergencia tecnocientífica nano-bio-info-cogno
}

JAMER EQHEVERIA*

\section{Resumen}

La emergencia de la tecnociencia en el siglo XX ha transformado la práctica científica y también el concepto de interdisciplinariedad. Este artículo analiza un ejemplo relevante de tecnociencia contemporánea, el Informe Converging Technologies for Improving Human Performance (CTIHP) de la National Science Foundation (NSF) norteamericana, en el que se promueve un nuevo tipo de interdisciplinariedad, nano-bio-info-cogno (NBIC). El análisis del informe de la NSF muestra que el concepto de interdisciplinariedad que se usa es instrumental, selectivo y, a veces, parece ser reduccionista, en perjuicio de las ciencias sociales, las artes y las humanidades. Como tesis principal, se sugiere que la noción de interprofesionalidad es más adecuada para la tecnociencia que la de interdisciplinariedad.

Palabras clave: Interdisciplinariedad. Convergencia tecnológica. Nano-bio-infocogno tecnociencia. Interprofesionalidad.

\footnotetext{
* Investigador Ikerbasque, Departamento de Sociología 2, U niversidad del País Vasco. Profesor de Investigación, Instituto de Filosofía, CSIC, Madrid, España.

1 Este artículo ha sido elaborado en el marco del proyecto de investigación HUM 2005-02105/FISO, "Racionalidad axiológica de la práctica tecnocientífica", financiado por el M inisterio de Educación y Ciencia del Gobierno de España durante el período 2005-2008. La parte final fue redactada durante una estancia investigadora en la U niversity of N evada, Reno (CBS, M atheson Knowledge Center) en otoño de 2008.
} 


\section{Emergencia y desarrollo de la tecnociencia en la segunda mitad del siglo XX.}

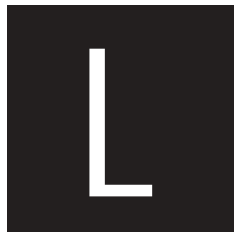

os programas Converging Technologies muestran casi todas las características que distinguen la tecnociencia contemporánea de la ciencia moderna. La ciencia pretende ante todo conocer cómo es el mundo: describirlo, interpretarlo, comprenderlo, explicarlo y, en el mejor de los casos, predecir a priori los sucesos que vayan a ocurrir y retrodecir lo que sucedió, explicándolo a posteriori. El objetivo principal de la tecnociencia, en cambio, consiste en transformar el mundo, sea éste natural, social o artificial. A la tecnociencia le interesa el conocimiento, por eso tiene una componente científica, pero con una diferencia importante: para un científico el conocimiento es un fin en sí, mientras que para un tecnocientífico es un medio para lograr objetivos de otra índole. A quienes promueven y financian la tecnociencia no les basta con la investigación que genera conocimiento. Además, pretenden que de dicha investigación surjan desarrollos tecnológicos y, más recientemente, también innovaciones. Por eso se empezó a hablar en el siglo XX de los sistemas de investigación y desarrollo $(I+D)$, y a finales de siglo de los sistemas de $I+D+i$, añadiendo al final la innovación, que ha pasado a convertirse en el objetivo último de las actividades tecnocientíficas, las cuales son más complejas y diversas que la investigación científica.

La tecnociencia surgió en la Segunda Guerra M undial y en una primera fase estuvo impulsada por los grandes programas de investigación que, financiados por el gobierno de los EEUU, conformaron la Big Science en dicho país (SO LLA PRICE 1955). Dicha emergencia coincide con el momento en que apareció la política científica y en el que se configuró el sistemas estadounidense de $I+D$, estrechamente vinculado a las empresas y al sector industrial, así como a instituciones militares. La ciencia académica, 
en la medida en que no dio el paso hacia la investigación aplicada y la transferencia de conocimiento al sector productivo, fue perdiendo relevancia. Así surgió la ciencia postacadémica (Ziman), a la que por varios motivos es preferible denominar tecnociencia (Latour). La Big Science estuvo desde su origen estrechamente vinculada a las empresas industrialesy a organizaciones militares (proyectos Manhattan, ENIAC, Radiation Laboratories, nuevos materiales, medicina de guerra, nuevos métodos de organización de los ejércitos, etc.). En esta época se estableció el Ilamado contrato social de la ciencia, que consiste en una alianza estratégica entre científicos, ingenieros, técnicos, empresarios, industriales, políticos y, en muchos casos, militares. Conjuntamente, estos siete diferentes tipos de agentes componen la agencia tecnocientífica, que se ha organizado durante el siglo XX en losEEU U en base a los macroprogramas de investigación de la Segunda Guerra M undial y a iniciativas similares en la época de la guerra fría (navegación espacial, escudos antimisiles, telescopio espacial H ubble, lucha contra el cáncer en la época de $\mathrm{N}$ ixon, etc.). La agencia tecnocientífica está compuesta por una pluralidad de agentes que se han ido aglutinando en torno a esos macroprogramas de investigación. Cada uno de esos agentes, ciertamente, está interesado en el conocimiento científico, pero desde muy diferentes perspectivas. Los científicos creen que es un bien en sí y pretenden ante todo lograr avances en el conocimiento, haciendo investigación básica para ello y publicando los resultados en las revistas especializadas de cada disciplina. Los ingenieros y técnicos también están organizados disciplinariamente, y se interesan en el know how y en los desarrollos tecnológicos que surgen de la investigación, tanto básica como aplicada: mejorar la eficiencia de los sistemas técnicos y solucionar problemas son sus objetivos prioritarios. Algunos empresarios e industriales vieron en la Big Science importantes oportunidades para crecer y obtener beneficios, así como para incrementar su nivel de competitividad y ampliar sus cuotas de influencia en el mercado: muchos de ellos tuvieron 
éxito, por lo que la investigación científica comenzó a ser promovida por dichas empresas, insertándose esos laboratorios en complejos de producción industrial. Por su parte, las instituciones militares más importantes tuvieron claro desde la primera Guerra M undial (la llamada guerra química), que los avances científico-tecnológicos son decisivos para generar nuevas armas, tanto para el ataque como para la defensa; otro tanto cabe decir de la medicina y la curación de las heridas y enfermedades de una época de guerra. La alta valoración militar de la ciencia y la tecnología se vio confirmada todavía másen la segunda Guerra M undial, así como en los demás conflictos bélicos del siglo XX y en la Guerra Fría entre losEEUU y la URSS, buena parte de la cual fue una contienda tecnocientífica (armas nucleares, exploración espacial, redes electrónicas defensivas, sistemas de misiles, servicios de información, espionaje industrial, etc.). En suma, los grandes ejércitos han tenido claro durante el siglo XX que los progresos de I+D son decisivos para la Defensa, por lo que crearon sus propios laboratorios de investigación e impulsaron y desarrollaron diversosmacroproyectos de investigación secretosy de importancia estratégica. En cuanto a los políticos, al promover esos grandes programas de investigación satifficieron a numerosos lobbies de presión, por una parte, y por otra impulsaron el crecimiento económico de los EEUU (y de la URSS). LaS grandes inversiones públicas en I+D fueron una locomotora económica para muchas industrias y empresas, precisamente porque dichos programas eran, por así decirlo, interdisciplinares, esdecir, incidían en variasáreas de conocimiento, y también en diversas disciplinas técnicas. Diremos pues, como primera propuesta de este artículo, que la Big Science fue la primera impulsora de la interdisciplinariedad, aunque en un sentido muy específico, que desborda la ciencia académica y la propia noción de disciplina. Argumentar esta hipótesises una de las pretensiones de este artículo.

Conceptualmente hablando, la Big Science estuvo y está basada en el modelo lineal del conocimiento, que fue formulado por primera vez por 
Vannevar Bush en su célebre informe al presidente Roosevelt, Science, the Endless Frontier(1945). A nuestro juicio, dicho documento aporta una primera teorización de la tecnociencia, entendida ésta como un cambio en la estructura de la práctica científica (Echeverría 2003). Dicha transformación se concretó en los años 50 y 60 en los macroproyectos de investigación y en el ya mencionado "contrato social de la ciencia", así como en la creación de nuevas agencias para impulsar la tecnociencia, como la National Science Foundation, Ios National Institutes of Health y la propia NASA. Por su parte, el Departamento de Defensa creó sus propias agencias de investigación tecnomilitar.

Pues bien, en dicho informe comienza a promoverse una nueva modalidad de interdisciplinariedad, que en primer lugar consistió en juntar a científicos e ingenieros y ponerlos a trabajar juntos cotidianamente en proyecto de investigación conjuntos; pero, además, en esos macroproyectos confluyeron también empresarios, políticos y militares, puesto que sus impulsores y, en último término, quienes financiaban las investigaciones, pero con objetivos muy concretos: fabricar la bomba atómica, crear el primer ordenador electrónico para aplicarlo al cálculo de trayectorias de bombasy a la simulación de explosiones, etc. V. Bush le subrayó una y otra vez a Roosevelt que los grandes avances científicos y tecnológicos que se habían producido durante la Guerra M undial se debían ante todo a que los científicos habían trabajado en equipo, es decir, a que la investigación no se había desarrollado de la manera tradicional, en los laboratorios de las universidades y centros de investigación (ciencia académica), sino en instituciones de nuevo cuño, creadas durante la guerra, que a nuestro juicio aportaron una nueva organización de la práctica científica. El laboratorio de Los Alamos es el ejemplo más típico, pero hubo otros muchos. O bligados por las circunstancias, muchos científicos habían colaborado activamente con instituciones militaresy organizaciones industriales ajenas hasta entonces a sus propias comunidades disciplinarias. Pues bien, uno de los principales 
consejos de Vannevar Bush al Presidente Roosevelt unos meses antes de que terminara la Segunda Guerra M undial con la victoria estadounidense, consistió en mantener la misma estructura de "trabajo en equipo", como Bush la denominó en su Science, the Endless Frontier. Se vislumbra así un nuevo concepto de interdisciplinariedad, que no sólo afecta a las disciplinas científicas, tal y como éstas se organizan académicamente, sino a una división más amplia del trabajo: la que permite distinguir entre diversas profesiones y sectores sociales, con sus respectivas tradiciones culturales (científicos, ingenieros, empresarios, políticos y militares, en nuestro caso). Esa organización intersectorial de la actividad tecnocientífica va a ser la dominante en los actuales programas Converging Technologies Nano-Bio-InfoCogno (NBIC), por eso dichos programas son un canon de la tecnociencia.

Esa nueva organización "interdisciplinaria” de la investigación también se puso en práctica, por cierto, en la elaboración del propio informe Science, the Endless Frontier. Aunque el informe final lo redactó el propio Vannevar Bush, previamente se habían reunido numerosos comités y grupos de expertos para asesorar a Bush en relación a la pregunta que le había formulado Roosevelt: ¿cómo organizar en tiempos de paz la impresionante maquinaria de I+ D que los EEUU habían creado durante la guerra? Esimportante señalar que en el momento en que surge la propia noción de política científica y se diseña el sistema norteamericano de I+D, quienes hicieron ese diseño no fueron científicos de una determinada disciplina, sino de varias; y ni siquiera ellos solos, puesto que también intervinieron otro tipo de expertos: responsables políticos y militares, representantes de grandes empresas, etc. El modelo de agente que diseñó la política científica en 1945 y la que ha diseñado en 2000 y 2001 los programas Converging Technologies NBIC es básicamente el mismo: por una parte interdisciplinario, por otra parte interprofesional. A ese tipo de agente de la tecnociencia, que no ha existido como tal en la época de la ciencia moderna, lo denominamos 
agencia tecnocientífica. Esimportante subrayar que no sólo impulsa y lleva a cabo la investigación científica, sino que también la diseña, proponiendo objetivos que no se limitan a los avances en el conocimiento, sino señalando hitos tecnológicos e innovaciones que se trataría de alcanzar en pocos años. La emergencia de la tecnociencia es simultánea a la de las políticas científicas y los microprogramas de investigación, siendo la tarea de diseño netamente interdisciplinar. Aquí no insistiremos más en este punto, que merecería un estudio histórico detallado en los diversos países, analizando en qué momento surgieron las políticas científicas en cada país, y quién las impulsó.

En todo caso, el trabajo conjunto en los laboratorios militares de científicose ingenieros durante la década de los 40 , y luego en la postguerra, supuso una primera forma de interdisciplinariedad ampliada, puesto que se forzó a colaborar activamente a representantescualificados de dosgrandesculturas del conocimiento, la científica y la ingenieril, que tradicionalmente habían sido independientes entre sí, aun habiéndose influido mutuamente. Pero la noción de "trabajo en equipo" que utilizaba V. Bush no sólo se refería a esa colaboración sistemática entre científicos e ingenieros en el marco de proyectos y equipos conjuntos, sino ante todo a una convergencia entre culturas y sectores sociales más amplios y más diversos, como los anteriormente mencionados. En suma, nuestra primera propuesta afirmar que el llamado "contrato social de la ciencia" puede ser interpretado como una nueva concepción de la interdisciplinariedad, que desborda los límites de la ciencia académica.

La noción de disciplina tiene una raigambre muy antigua, que se remonta a las universidades medievales, y por tanto es netamente académica. El contrato de Bush vincula estrechamente a académicos, técnicos, hombres de negocios, políticos y expertos en estrategias de seguridad y defensa. Implica una interdisciplinariedad ampliada, que en realidad rompe con la propia noción de interdisciplinariedad, porque ésta depende de las diversas formas de cultivar el conocimiento, mientras que en la tecnociencia sólo se cultiva el conocimiento para ponerlo ulteriormente en valor (económico, 
empresarial, militar, político) y lograr objetivos de índole no científica (políticos, militares, económicos, industriales, estratégicos, etc.). Insistimos de nuevo: para la ciencia el conocimiento es un fin, para la tecnociencia no es más que un medio. La interdisciplinariedad no es más que un modo de organizar mejor (o peor) el trabajo en equipo, bien entendido que dicho trabajo consiste en investigar y producir conocimiento. Lo importante es la convergencia de profesiones, sectores sociales y culturas diferentes en una misma actividad, que se supone que va a aportar beneficios a todos y cada uno de los participantes en ella, siendo esos beneficios de índole muy distinta: epistémicos, políticos, militares, empresariales, sociales, etc.

La Big Science sigue existiendo hoy en día, pero a partir de los años 80 emergió una nueva modalidad de tecnociencia, promovida principalmente por la iniciativa privada en EEUU. Varios fueron los factores que influyeron en esa evolución, pero cabe mencionar dos muy relevantes: una nueva política fiscal promovida por la administración Reagan, y cambios significativos en la ley estadounidense de Patentes. A partir de ese momento, surgieron expectativas importantes de que la inversión en I+D fuera rentable para los inversores, lo que atrajo mucho dinero privado a las Bolsas, invirtiendo en empresas de I+D. Asimismo surgió una nueva modalidad de entidad financiero, las empresas de capital-riesgo, buena parte de cuyas inversiones se destinaron a pequeños proyectos tecnocientíficos con alto potencial innovador. El éxito del nuevo modelo fue inmediato, particularmente en el sector de las TIC, farmacología, tecnomedicina y tecnociencias de la alimentación. Empresas como Apple, Microsoft, Sun, Cisco, M onsanto, Google, Nokia, etc., ejemplifican bien esta segunda fase de la tecnociencia, que tiene varias diferencias estructurales con la Big Science que aquí no comentaremos. Nos limitaremos a mencionar dos:

- La importancia creciente de la innovación, que en primera instancia ha dado lugar a la emergencia de los sistemas de I+ D+ i y posteriormente ha generado sistemas locales, regionales y nacionales de 
innovación (LUNDVALL, 1992). Cabe afirmar que hoy en día los sistemas de innovación constituyen el motor principal de las economías. Como resultado de este proceso, que ha tenido lugar en los últimos 25 años en numerosos países, el conocimiento y la innovación se han convertido en "las nuevas fuentes de riqueza, poder y calidad de vida" (M. CASTELLS, 2007, p. 20). Ello define la segunda etapa de la tecnociencia, caracterizada por la proliferación de pequeñas y medianas empresas tecnocientíficas que, al ser altamente innovadoras, han acabado generando importantes empresas y consorcios, como los anteriormente mencionados.

- La estrecha vinculación entre esta segunda modalidad de tecnociencia y el desarrollo de las sociedades de la información y el conocimiento, que se contraponen a las formas de producción industrial típicas de la Big Science. Así como la ciencia y la ingeniería fueron muy importantes para las empresas industriales, las empresas tecnocientíficas privadas que han proliferado en el último cuarto de siglo se caracterizan por el uso intensivo de las tecnologías de la información (TIC) como medio relevante para producir conocimiento (e-science, arte digital, música electrónica, etc.), pero también para distribuirlo, difundirlo, comercializarlo y utilizarlo.

La aparición de lasempresas tecnocientíficas a partir de 1980 ha implicado una superación radical de las antiguas fronteras disciplinarias, en particular por la importancia que han tenido las TIC como fuente de innovación. Las acciones de casi todos los agentes relevantes en la economía de la información y el conocimiento están mediatizados por el sistema tecnológico TIC, cuyo buen funcionamiento depende de múltiples disciplinas científicas e ingenieriles, pero también de otrosfactores ajenos a la ciencia y la ingeniería clásicas. Cualquier investigador científico, salvo raras excepciones, tiene que utilizar hoy en día dichos instrumentos tecnocientíficos, cuyo diseño, fabricación y uso implica la implementación de varias modalidades de 
conocimiento. Las TIC son interdisciplinarias por su propia constitución y diseño, y se han difundido prácticamente por todos los sectores sociales. Por tanto, la emergencia de la tecnociencia privada rompió nuevamente las fronteras disciplinarias, propiciando la aparición de equipos en donde los ingenierosy técnicos colaboran con científicos, artistas, músicos, arquitectos, etc., con el fin de que éstos últimos puedan llevar a cabo sus actividades con ayuda de las TIC. Por otra parte, cada una de las disciplinas científicas se ha visto profundamente transformada en su práctica cotidiana en los últimos años, hasta el punto de que cabe distinguir hoy en día entre matemáticas y tecnomatemáticas, física y tecnofísica, biología y tecnobiología, medicina y tecnomedicina, etc. Por cierto, también cabe distinguir entre artes y tecnoartes, humanidades y tecnohumanidades y ciencias sociales y tecnociencias sociales. Esas nuevas prácticas y formas de generar conocimiento han traído consigo un impulso muy fuerte hacia esa interdisciplinariedad ampliada de la que hablamos, tendencia ésta que no parece tener retorno. Para describir de manera sintética este cambio diremos que la tecnociencia no la hacen individuos ni personas singulares, algo que sí sucede con la ciencia, sino ante todo grupos, equipos, empresas y agencias, siendo estas agencias colectivas e interdisciplinares, es decir, reuniendo a varias personas de diferentes procedencias disciplinarias y profesionales y poniéndolas a colaborar en proyectos conjuntos regidos por el imperativo de innovar. Desde la perspectiva de análisis que estamos proponiendo, la interdisciplinariedad se convierte en un modo de organización del trabajo que favorece la innovación, siendo tanto más valiosa cuanta más innovación suscita. En suma: en la tecnociencia actual la interdisciplinariedad es un medio para incrementar la capacidad de innovación de los grupos que investigan y generan conocimiento, pero también de los que lo distribuyen, difunden y utilizan.

Esta será nuestra segunda propuesta en este artículo. U na vez enunciada, vamos a comentar las dos propuestas con mayor detalle, antes de pasar a ejemplificarlas en el caso de los programas Converging Technologies NBIC. 


\section{Producción de conocimiento e interdisciplinariedad.}

Gibbons y otros autores publicaron en 1994 un libro titulado The New Production of Knowledge, en el que afirmaban que la investigación científica había cambiado durante lasúltimas décadas, pasando del modo 1 al modo 2 de producción de conocimiento. El modo 1 tiene lugar en un contexto académico y esdisciplinario, homogéneo, jerárquico y estable. La jerarquía académica tiene a su cargo el control de calidad del conocimiento producido, por ejemplo mediante el peer system review y la selección de las personas que van a ser reconocidas como investigadores profesionales. Por tanto, en el modo 1 la comunidad científica determina qué conocimiento es válido y cuál no, en función de sus propios criterios de valoración. En cambio, en el modo 2 la investigación no sólo está impulsada por los científicos, sino por varios agentes. Desde el diseño de los proyectos se tienen en cuenta sus posibles aplicaciones futuras, no sólo el conocimiento que se va aportar a una determinada disciplina. El modo 2 de producción de conocimiento es transdisciplinario, heterogéneo, heterárquico y variable. La calidad del conocimiento generado la valoran diferentesagentes, en función de diversos criterios. Por otra parte, loscientíficosse sienten responsables ante la sociedad que financia sus investigaciones, siempre según Gibbons.

Todo ello es cierto, y tiene mucho que ver con la tecnociencia de la que estamos hablando, pero la distinción entre dos modos de producción de conocimiento resulta insuficiente, a nuestro modo de ver, ante todo por la razón siguiente: en la economía del conocimiento y, en su caso, en las sociedades del conocimiento, tan importante es la producción del conocimiento como luego su distribución, aceptación y utilización. A nuestro modo de ver, la tecnociencia no sólo afecta a los modos de producción del conocimiento, también al resto de sectores de la economía del conocimiento. Por tanto, nuestra propuesta amplía la de Gibbons y tiene en cuenta la transformación que se ha producido en el conjunto de la práctica científica, no sólo en la investigación (ECHEVERRÍA, 2003). La actividad científica no se 
reduce a la investigación, esmucho másamplia. Un informe como el de Vannevar Bush no lo hace una persona, sino una comisión interdisciplinaria de expertos, aunque al final haya alguien que dé nombre al documento. También son grupos de expertos quienes diseñan las políticas científicas, previos debates, discrepancias y procesos de consenso. Además, la tecnociencia también transforma los modos en los que las personas, los grupos, las empresas y las instituciones se apropian de las innovaciones de origen científico y lasutilizan, como el ejemplo de las TIC deja claro. Podríamos hablar, por tanto, de un modo 1 y un modo 2 a la hora de evaluar el conocimiento, de utilizarlo o de distribuirlo, no sólo de producirlo. Las propuestas de Gibbons van en la buena dirección, pero están demasiado centradasen la investigación, siendo así que la actividad científica incluye otrostipos de acciones, al igual que la tecnológica. Por todo ello preferimosutilizar el término 'tecnociencia' que propuso Latour para interpretar esta gran transformación, entendiendo el paso de la ciencia a la tecnociencia como un cambio en la estructura de la práctica científica que afecta a cada una de sus fases: planificación, producción, evaluación, difusión y aplicación del conocimiento, sin olvidar la enseñanza de la ciencia, que resulta clave en cualquier sistema científico-tecnológico. La tecnociencia se distingue de la ciencia por su modo de relacionarse con el conocimiento en cada una de esas fases, no sólo en la de investigación y producción del conocimiento, que tradicionalmente ha sido la más estudiada.

En realidad, pensamosque los científicosy los ingenieros involucrados en la actividad tecnocientífica son, ante todo, trabajadores del conocimiento (DRU CKER, 1994). En tanto trabajadores de dicho sector, han sido contratados al servicio de las empresas tecnocientíficas, sean éstas privadas, públicas o mixtas, incluyendo las instituciones y organizaciones tecnomilitares. Si ello es así, los científicos e ingenieros no se sienten responsables ante la sociedad en general, sino más concretamente ante los agentes que financian sus investigaciones, porque son quienes les pagan y porque sus tareas y obligaciones han sido especificadas en sus contratos con dichas empresas 
tecnocientíficas, que son los que tienen que cumplir como trabajadores del conocimiento. Para ello, los científicos e ingenieros han de interiorizar en sus líneas de investigación los valores y objetivos de la agencia o empresa tecnocientífica de la que dependen, 0 al menos acatarlos y comportarse conforme a los diseños previos de las líneas prioritarias de investigación para dichas agencias o empresas. Por ser trabajadores del conocimiento no renuncian a sus objetivosy valores epistémicos, por ejemplo a lograr avances en el conocimiento y hacerlos públicos, conforme al ethos de la ciencia (M ERTO N). Lo que ocurre es que, en muchas ocasiones, quedan subordinados a otros valores y objetivos, no en vano los programastecnocientíficosy sus líneas prioritarias están diseñados previamente, en la mayoría de los casos con gran detalle.

Este cambio en la estructura de la práctica científica no ha sido asumido por muchos científicos, que siguen manteniendo su sistema de valores académicos tradicionales y se dan por satisfechossi generan avances en el conocimiento y sus aportaciones son reconocidas por su comunidad disciplinaria. Algunos, en cambio, sí lo han asumido. Denominaremos científicos a los primeros y tecnocientíficos a los segundos, dejando claro que en algunas ocasiones la frontera entre ambos es difusa. En términos de Gibbons y sus colaboradores, los primeros producen conocimiento según el modo 1 , los otros conforme al modo 2. A nuestro modo de ver, también cabría distinguir entre quienes evalúan, difunden o aplican el conocimiento según el modo 10 el 2, como acabamos de mencionar.

En el caso de las empresas privadas las cosas suelen estar mucho más claras, porque han sido diseñadas desde la perspectiva de la tecnociencia y la innovación, a diferencia de muchas universidades y centros de investigación públicos, que mantienen idearios correspondientes a las tradiciones de la ciencia moderna. Por otra parte, la emergencia de la tecnociencia puede caracterizarse también, desde una perspectiva presupuestaria, por el predominio de la financiación privada sobre la pública. Aunque haya 
tecnociencia de financiación pública que mantiene otros objetivosy sistemas de valores, la mayor parte de la tecnociencia actual (casi el $80 \%$ en EEU U y Japón) está financiada por el sector privado. Por tanto, opera conforme a modelos de gestión del conocimiento que nada tienen que ver con los criterios académicos tradicionales. En tales casos el concepto clásico de interdisciplinariedad apenas es relevante. Lo que importa es que cada trabajador del conocimiento contratado por la empresa tecnocientífica realice satisfactoriamente la tarea que le ha sido asignada. La interdisciplinariedad tecnocientífica tiene que ver con la tradicional división del trabajo, pero referida a las economías de la información y el conocimiento.

\section{Los programas Converging Technologies.}

La investigación científica a escala nanométrica comenzó en los años 80 del siglo XX. Hubo una etapa básicamente científica (1980-2000), que conviene distinguir de la etapa tecnocientífica. Esta segunda etapa empezó el año 2000 cuando el Presidente Clinton anunció la National Nanotechnology Iniciative (N NI) de losEEUU, que fue aprobada por la Cámara de Representantes y el Senado. Diversos agencias federales y lobbies tecnocientíficos, incluidas instancias militares, habían presionado desde 1996 para que EEU U diseñara una política científica ambiciosa en relación a las nanotecnologías. La N N I sentaba las bases para ello, al atribuir a la N ational Science Foundation el cometido de diseñar una estrategia y proponer un macroprograma para impulsar la I+D + i en el ámbito de las nanociencias y las nanotecnologías, a las que nosotros denominaremosnanotecnociencias (NTC), por no ser sino una nueva modalidad de tecnociencia aplicada a los nanocosmos (escala $10^{-9} \mathrm{~mm}$.).

La posibilidad de estudiar el mundo a escala tan pequeña había sido augurada en 1959 por Richard Feynman, Premio Nobel de Física, en su célebre conferencia "There's plenty of room an the bottom", en la que 
imaginó la posibilidad de una ingeniería a nivel atómico (FEYN M AN, 1961). Pero esa premonición tuvo visos de realidad sólo a partir de 1981, cuando Gerd K. Binnig y Heinrich Rohrer diseñaron y construyeron el microscopio de efecto túnel (scanning tunneling microscope, STM), avance por el que ganaron el Premio N obel de Q uímica. D icho aparato permitió a los investigadores representar cada átomo concreto. Pocos años después se produjo otro gran avance, también de índole tecnológica: Sumio ljima inventó los nanotubos de carbón, cuya utilidad en el desarrollo de las NTC ha sido enorme. Por su parte, Warren Roninet y R. Stanley Williams crearon un sistema de realidad virtual conectado a un microscopio de efecto túnel. Esto y la invención previa del STM supusieron pasos decisivos, puesto que permitieron "ver" los átomos (es decir, simularlos con ayuda de poderosos ordenadores) y ulteriormente operar con ellos, modificando su posición relativa y generando, por tanto, átomos y moléculas nanotecnológicamente modificados. A partir de ese momento las ingenierías a nivel atómico, molecular y celular comenzaron a ser posibles: los seres humanos aprendieron a conocer un ámbito del mundo que hasta el momento había sido inaccesible para ellos, y simultáneamente a transformarlo. Desde entonces, el afán por conocer cómo son los macrocosmos casi siempre ha estado subordinado a la voluntad de modificarlos y transformarlos.

De inmediato surgieron instituciones y empresas dedicadas a las nanotecnologías, tanto en EEU U como en Europa y otros países. Asimismo se empezó a investigar otras posibles implementaciones de las nanotecnociencias mediante artefactos TIC, cosa que resultó posible cuando en 1996 un grupo investigador de la D elf U niversity of Technology (H olanda) creó un transistor usando nanotubos de carbón. A partir de ese momento, se abrió la posibilidad de que las nanotecnologías y las TIC convergieran en otros muchosámbitos, idea que está en el origen de los programas Converging Technologies. Por su parte, la Comisión Europea hizo publica en 1998 la 
iniciativa N ID (Nanotechnology Information Devices), dentro del programa Information Society Technologies. Las expectativas que abría la convergencia Nano-Info ya entonces eran considerables, luego no han hecho más que aumentar, en particular en lo que se refiere a la miniaturización de los chips. Conviene destacar que en 1999 el Departamento norteamericano de Defensa estableció que las nanotecnologías conformaban un área de importancia estratégica, lo que tuvo gran importancia para su futuro inmediato, en primer lugar porque dicho Departamento creó sus propios laboratoriosy centros de investigación, y desarrolló sus propios programas, pero, además, porque a partir de ese momento determinados resultados de la actividad investigadora pasaron a ser confidenciales y secretos.

Cuando los Lucent \& Bell Laboratories y la Universidad de O xford crearon en 2000 el primer motor de DNA con ayuda de las NTC, se abrió una nueva posibilidad de convergencia, en este caso entre las biotecnologías y las nanotecnologías. Con todos esos pasos previos, más la aprobación de la National Nanotechnology Initiative, se daban todas las condiciones para que la N ational Science Foundation (NSF) norteamericana diseñara e hiciera público en 2001 un macroprograma de investigación denominado Converging Technologies for Improving Human Performance (CTIH P), y habitualmente conocido como Convergencia NBIC (nano-bio-info-cogno), puesto que dicha iniciativa pretende interconectar estrechamente las líneas de investigación en esos cuatro grandes ámbitos de la tecnociencia contemporánea.

No entraremos a comentar los múltiples puntos relevantes de dicho programa. En este artículo nos limitaremos a comentar sus implicaciones en relación a la interdisciplinariedad, que son muchas. La comisión de expertos que diseñó el extenso documento publicado por la NSF (ROCO; BAIN BRIDGE, Converging Technologies, 2001) estuvo compuesta por 70 personas, de las cuales 37 provinieron del gobierno federal o de laboratorios nacionales, 26 eran académicos, representando a diversas universidades 
norteamericanas, y 17 representaban al sector privado, es decir, a lasprincipales empresastecnocientíficas privadas. Al menos cuatro de losparticipantes venían del Departamento de Defensa y 6 de la NASA y otros organismos federales de Aeronáutica. En resumen: dicho Comité se adecuaba bien a la estructura de lo que anteriormente hemos definido como agencia tecnocientífica. Brillaban por su ausencia los representantes de la sociedad civil, porque ni siquiera había parlamentarios, sólo expertos en política científica, en particular de la NSF, que fue la institución más representada. Dos de sus miembros más destacados, M ihail Roco y W illiam S. Bainbridge, se encargaron de redactar el informe final, que casi llega a 500 páginas y da cuenta del trabajo de los numerosos paneles de trabajo en los que se subdividió la Comisión. También fueron ellos dos quienes editaron un segundo informe sobre las implicaciones sociales de una iniciativa de tanta envergadura (RO CO ; BAIN BRIDGE, Societal Implications, 2001). En este caso la Comisión que elaboró el informe estuvo formada por 64 personas, de las cuales 17 representaban al sector privado, 28 a las instituciones académicas y 21 al Gobierno Federal y sus Agencias de investigación, incluida nuevamente la NASA, pero sin representante alguno del Departamento de Defensa, lo cual resulta también significativo por lo que respecta a las relaciones entre los Ejércitos y la sociedad.

En ambos informes estamos nuevamente ante la interdisciplinariedad ampliada de la que antes hablamos. La representación de la academia fue muy pluridisciplinar, puesto que un programa así incide en las más diversas áreas de conocimiento; pero lo más destacado es que entre empresarios, expertos en política científica y representantes de instituciones militares y agencias gubernamentales había un número superior al de académicos, y ello en ambos Comités. Los científicos e ingenieros tienen un peso significativo a la hora de diseñar los grandes programas tecnocientíficos, pero hay otras muchas instancias que intervienen en dicho diseño y, sobre todo, en la toma final de decisiones, en particular las relativas a la financiación de cada acción y línea del programa. Esas decisiones suelen escapar al ámbito de 
decisión de los científicos, cuyo papel suele ser consultivo; a no ser, claro está, que un científico se convierta en un político o en un empresario profesional, sin dejar de apelar a su condición de científico, pero desempeñando una función de gestión del conocimiento. Esta modalidad de interdisciplinariedad esmuy frecuente en lastecnociencias contemporáneas. Cumplida una cierta edad, es habitual que muchoscientíficos dediquen buena parte de su tiempo de trabajo a la evaluación y la gestión del conocimiento, y en concreto a la participación en comités interdisciplinarios de asesoramiento. En el caso del Comité de la NSF, hubo varios muy destacados, por ejemplo John Watson, quien formó parte del Comité que diseñó el programa CT como representante del National Institute of Health. Este tipo de personas siguen siendo científicos, pero ya no hacen investigación, sino gestión del conocimiento, o también política científica. Su formación no ha cambiado, pero su profesión sí. No se trata de un cambio de disciplina académica, sino de algo muy distinto, y muy típico de la tecnociencia: el científico comienza a practicar una nueva profesión, por ejemplo la dirección y gestión de importantes programas y centros de investigación, como ha sido el caso de W atson en las últimas décadas (proyecto Genoma en su primera fase).

No es lo mismo ser un investigador científico que un gestor del conocimiento, un profesional de la política científica o un difusor de la ciencia, sea como docente o como divulgador. Hemos mencionado hasta cinco profesiones distintas para un mismo científico, y en todos los casos se está trabajando con el conocimiento. Lo que resulta insuficiente es reducir la esfera del conocimiento a su producción, sin tener en cuenta que la actual economía del conocimiento esmucho más compleja, como en su momento señaló D rucker (DRU CKER, 1998). Por eso introducimosla noción de interprofesionalidad de la tecnociencia, distinguiéndola de la interdisciplinariedad científica, que también se da en los Converging Technologies.

M as retomemos el desarrollo histórico de los programas Converging Technologies. La U nión Europea creó en 2003 su propio grupo de expertos, que publicó en junio de 2004 un informe titulado Converging Technologies 
for the European Knowledge Society (A. NORDM ANN, CTEKS, 2004). La Comisión Europea también encargó y publicó un informe sobre los posibles riesgos asociados (CE, Preliminary Report, 2004). El procedimiento de elaboración también se basó en los grupos de expertos, aunque las diferencias en la composición de ambos grupos fueron significativas: la mayoría de miembros de la comisión europea provenían de las ciencias socialesy, aunque había representantes del sector empresarial, no hubo nadie que ocupara cargos en la Comisión ni tampoco representantes de instituciones militares. Por tanto, la Comisión de expertosnombrada por la U Eera bastante interdisciplinaria en el sentido tradicional de la palabra, pero apenas satisfacía el criterio de interprofesionalidad recién mencionado. De hecho, su informe ha tenido una influencia y repercusión mucho menor que el estadounidense. Losprocesos de toma de decisiones con respecto a las políticas de convergencia tecnocientífica han ido en la UE por otras vías, dado el gran peso específico que tienen los Estados en las decisiones finales.

Tanto el programa europeo como el norteamericano propugnaron la convergencia entre cuatro grandes sistemas tecnológicos: las nanotecnologías, las biotecnologías, las tecnologías de la información y las ciencias cognitivas. Ello implica un impulso claro hacia la interdisciplinariedad. En el caso europeo, también se promovió, aunque sólo fuera a nivel de declaración de principios, la convergencia de las disciplinas NBIC con algunas ciencias sociales, por ejemplo la sociología, la antropología e incluso la filosofía. O tros muchos países (Canadá, China, Japón, Sudáfrica, Brasil, Australia, Rusia, etc.) están desarrollando iniciativas similares, habría que estudiar en concreto si sus programas favorecen o no la interdisciplinariedad, y cómo lo hacen, pero en principio es de prever que así suceda, dada la tendencia general de esta main stream de la investigación tecnocientífica actual.

Dicha convergencia científica y tecnológica, de llevarse a cabo, podría cambiar considerablemente la noción de conocimiento. Para investigar es- 
tas cuestiones la interdisciplinariedad resulta indispensable, por eso lasciencias cognitivas están incluidas en el núcleo duro de dichos programas. El norteamericano, por ejemplo, diseña una línea de acción con el rótulo "Conquista de la Mente", ni más ni menos. No es de extrañar luego que los ciudadanos normales desconfíen de muchas tecnociencias cuando se eligen denominaciones así, particularmente agresivas. El programa europeo también promueve la convergencia entre disciplinas, pero por otros motivos: su objetivo principal consiste en favorecer el desarrollo de la sociedad europea del conocimiento, conforme a la Agenda de Lisboa 2000. En todo caso, en los dos programas la interdisciplinariedad es un medio para lograr mejor los principales objetivos de cada programa, los cuales no se cifran en generar conocimiento para conocer los nanocosmoso el cerebro humano, sino ante todo en que surjan desarrollos tecnológicose innovacionesque puedan mejorar lascapacidadeshumanas (en el caso estadounidense) o favorecer la emergencia y consolidación de la sociedad del conocimiento (en el caso europeo).

Podemos concluir que los programas Converging Technologies se corresponden perfectamente con las características que distinguen a las tecnociencias de las ciencias y las tecnologías. Además, fomentan la interdisciplinariedad, pero ante todo la interprofesionalidad, puesto que tanto su diseño como su ulterior implementación no sólo es obra de científicose ingenieros, sino ante todo de políticos, empresarios y militares, por mencionar los tres tipos de agentes que hay que añadir al "contrato social de la ciencia" de Vannevar Bush.

Para terminar, comentaremos algunos puntos del programa norteamericano en los que se alude expresamente a la necesidad de reformar los sistemas educativos y de formación promoviendo una mayor interdisciplinariedad, con el fin de que los programas Converging Technologies puedan desarrollarse adecuadamente. El fomento de la interdisciplinariedad en la tecnociencia parece llegar así a su máxima expresión, de ahí el interés 
de esta parte del informe y la atención que vamos a prestarle para analizar mejor la noción de interdisciplinariedad vigente en la tecnociencia.

\section{Reformar la educación para favorecer la convergencia nano-bio-info-cogno.}

Transformar la educación en ciencia e ingeniería, tanto a nivel universitario como desde la escuela primaria, es uno de los objetivos del informe norteamericano que, por eso es típicamente tecnocientífico, puesto que su objetivo consiste en la transformación del mundo, en este caso de los sistemas educativos norteamericanos. Dicho propósito se enuncia desde el principio, y aunque no es la primera prioridad, sí puede ser considerada como una de las principales. De hecho, el informe distingue seis major themes, titulándose el sexto U nifying science and education. El planteamiento de este sexto gran tema queda resumido del modo siguiente:

To meet the coming challenges, scientific education needs radical transformation from elementary school through postgraduate training. Convergence of previously separate scientific disciplines and fields of engineering cannot take place without the emergence of new kinds of people who understand multiples fields in depth and can intelligently work to integrate them. New curricula, new concepts to provide intellectual coherence, and new forms of educational institutions will be necessary. (ROCO ; BAINBRIDGE 2001, Converging Technologies, CTIHP, p. XI)

Tras haber sido anunciado así en el Executive Summary del Informe, parece que la propuesta de reforma educativa NBIC va en serio. La convergencia tecnológica nano-bio-info-cogno que se propugna y diseña, para ser llevada a cabo, requiere de una profunda transformación de los 
sistemas de formación científica e ingenieril. Según el informe, la formación NBIC habría de iniciarse en la escuela primaria y tener continuidad en todos los niveles educativos. Para ello habría que potenciar por doquier la interdisciplinariedad, aunque entendida en un sentido másparecido al de la interprofesionalidad antesmencionada que a la interdisciplinaridad académica, como a continuación intentaremos mostrar. En todo caso, conviene subrayar la enorme ambición transformadora que se manifiesta en este párrafo: se propone lisa y llanamente la reforma de todo el sistema educativo de ciencia y tecnología. El objetivo consiste en rediseñarlo de modo que favorezca al máximo el desarrollo del macroprograma tecnocientífico NBIC 0, dicho más claramente, en subordinar el sistema educativo a los objetivos de dicho programa. El programa norteamericano Converging Technologieses típicamente tecnocientífico porque no sólo incide en la investigación, sino en otras muchas actividades que tienen que ver con la ciencia y la tecnología, incluida la educación. De hecho, se plantea el objetivo de una transformación radical del sistema educativo norteamericano, pero no por razones pedagógicas, sino por objetivos e imperativo de otra índole, típicos de la agencia tecnocientífica de la que hablamosal principio de este artículo. No sólo se trata de transformar los nanocosmos, sino también de transformar los espacios de aprendizaje donde pueda enseñarse cómo son, y cómo pueden ser transformados.

Sin embargo, llama la atención la ausencia de ninguna concreción sobre cómo habría que planificar una reforma así de la educación de la ciencia y la tecnología. Cambiar un sistema educativo en todos sus niveles, como se pretende en el informe de la NSF, es una tarea muy compleja y difícil de lograr, como es bien sabido en el ámbito de las ciencias sociales y de las políticas educativas. Sin embargo, el informe CTIHP se limita a formular la propuesta y a afirmar las ideas que la inspiran, pero sin considerar objeciones, dificultadeso, simplemente, problemas legales o de financiación que pudiera acarrear una reforma de tal magnitud. Por decirlo en términos 
coloquiales, dicho informe entra en las cuestiones educativas "como elefante en cacharrería". Veámoslo brevemente.

Desde una perspectiva filosófica, llama la atención que una reforma educativa así tenga su fundamento en una noción, unifying science, que recuerda los mejores tiempos del positivismo y el empirismo lógico en la primera mitad del siglo XX, con su ideal de la ciencia unificada y la consiguiente elaboración de una Enciclopedia de la Ciencia U nificada para sentar las bases de dicha ciencia universal. Resurge así uno de los grandes mitos del reduccionismo en filosofía de la ciencia, la existencia de un lenguaje unificador de todas las ciencias, que ahora sería el lenguaje NBIC. Aunque la terminología que se utiliza en CHIHP es la de la convergencia y la interdisciplinariedad, el programa tecnocientífico que se presenta tiene un trasfondo fuertemente reduccionista y unificador. En último término, y contrariamente a la interdisciplinariedad o la transdisciplinariedad, el programa CHIHP implica un intento de reducción tecnocientífica de la pluralidad de las disciplinas científicasy tecnológicas a la unidad NBIC, supuesto que dicha unificación hubiera sido lograda. No sólo se trata de que diferentesáreas de la ciencia y la tecnología converjan, como reza el título del informe. Si se analiza su contenido más a fondo se comprueba que dicha convergencia está basada en la reaparición de una tentativa que parecía olvidada desde lostiempos del Círculo de Viena, la de unificar todas las ciencias en una única modalidad de ciencia, antes la ciencia fisicalista, ahora la ciencia NBIC. No insistiremos en esta crítica de índole filosófica al informe norteamericano, porque requeriría una extensa argumentación, pero sí conviene subrayar que la presunta interdisciplinariedad que se promueve resulta ficticia, dada la ideología unificacionista y reduccionista que subyace a buena parte del informe.

En esto se apoya todo el programa de reforma educativa que se propone. En el apartado $\mathrm{F}$ del informe, titulado Unifying Science and Education, y que fue elaborado por un panel compuesto por doce expertos, 
más el propio Roco como editor del informe, se afirma taxativamente lo siguiente:

Currently, scientific and engineering education is highly fragmentary, each part constrained by the boundaries of one particular discipline. In the future, the knowledge taught will be based on unifying concepts offered by nano, bio, info and cognitive sciences throughout the educational establishment. Natural, engineering, social and humanity sciences will converge. The corresponding basic concepts of unifying science will be introduced at the beginning of the teaching process in $\mathrm{K}-12$, undergraduate and graduate education (N SF, 2001, CTIHP, p. 363).

El informe combate decididamente la separación de las disciplinas científicas y tecnológicas y promueve su convergencia, y por ende la interdisciplinariedad, al menos aparentemente. Sin embargo, se trasluce una fuerte tendencia a la integración de la mayoría de las ciencias y tecnologías en un nuevo complejo tecnocientífico, todavía por elaborar, pero cuya existencia futura se quiere promover en las aulas desde los 12 años, e incluso antes. La tecnociencia NBIC, por designar en nuestrospropios términos a la ciencia unificadora a la que alude el informe, pasaría a ser el fundamento de casi todas las demás disciplinas científicas y tecnológicas, puesto que aportaría los conceptos básicos a todas ellas. Q ue sepamos, este gigantesco programa conceptual ni siquiera se ha emprendido (no está elaborándose ninguna Enciclopedia de la Tecnociencia Unificada), pero eso no parece importar mucho, porque la agenda tecnocientífica y educativa que se promueve queda expresada con toda claridad, así como su pretensión última de reformar por completo la enseñanza de las ciencias y las tecnologías. De nuevo estamos ante otra de las características de la tecnociencia: la agenda que finalmente se decide resulta ser más concluyente 
e importante que las razones o los contenidos por los cuales se adoptó, precisamente porque ello supone una decisión estratégica de la agencia tecnocientífica, cosa nada fácil de lograr. Si, además, el consenso es prácticamente total, como parece ser que ocurrió en el panel que tuvo a su cargo el tema de la educación y la ciencia unificadora, la agenda aprobada pasa a ser prácticamente incuestionable, casi un axioma de la tecnociencia. Por eso no hay necesidad de argumentarlo ni de probarlo, al menos internamente. Cuando haya que presentar esa agenda a la sociedad, en cambio, habrá que elegir muy bien los términos, imágenes y metáforas a utilizar. Para este último cometido se suele recurrir a las mejores agencias de publicidad científica disponibles, que cuentan con auténticos expertos en la elaboración de presentaciones política y socialmente correctas.

En todo caso, la tesis del informe CTHIP sobre educación es nítida y radical: "to enhance human performance most succesfully, science and engineering education will have to evolve and, in some respects, radically reinvent itself" (N SF, 2001, CTIHP, p. 365). Estamos ante un macroprograma tecnocientífico de gran envergadura, que no sólo pretende transformar la investigación en las áreas nano, bio, info y cogno, sino que apunta a objetivos de mucha mayor envergadura: ni más ni menos que replantear muchos aspectos de la educación científica y tecnológica, así como elaborar nuevas bases conceptuales para esa unifying science de la que se habla una y otra vez, y sobre cuyos principios básicos no se dice nada, salvo que debería emerger a partir del propio proceso de convergencia e integración de las diversas disciplinas en el complejo NBIC.

La elaboración del informe que estamos comentando vino precedida por un Simposio sobre el impacto social de la convergencia NBIC, cuyas actas también fueron editadas por Roco y Bainbridge en el segundo informe de la NSF, Societal Implications, ya mencionado. En este segundo informe se alude más explícitamente a la cuestión de la interdisciplinariedad, 
por lo que terminaremos esta contribución comentando algunos pasajes al respecto. A nuestro modo de ver, el segundo documento de la NSF aclara mucho las razones por las que la convergencia tecnológica propuesta exige esa gran reforma educativa:

The United States faces the daunting challenge of attracting enough of the best graduate students to the physical sciences and engineering disciplines. Under present conditions, far too few good students are attracted to the fields relevant to nanotechnology. To some extent, this is a problem faced by all of the sciences, but the problem is especially acute for nanotechnology because a very large number of talented scientists, engineers and technicians will be needed to build the nanotechnologies industries of the future, and these professionals will require an interdisciplinary perspective. Development of nanotechnology will depend upon multidisciplinary teams of highly trained people with backgrounds in biology, medicine, applied and computational mathematics, physics, chemistry, and in electrical, chemical, and mechanical engineering... Because current educational trends favour specialization, there must be fundamental changes in our educational systems. (ROCO; BAINBRIDGE 2001, Societal Implications, p. 13)

Por tanto, se constata en primer lugar un problema importante en las relaciones entre tecnociencia y sociedad: pocosjóvenes se sienten atraídos por las disciplinas que son relevantes para las nanotecnologías, y en general por las ciencias y las tecnologías. Ahora bien, esa "mano de obra futura" resulta indispensable para que la agenda CTIHP pueda desarrollarse, puesto que dicha agenda requiere la colaboración y contratación de personas con competencias y habilidades contrastadas en el ámbito nano-bio-info-cogno. 
Se requerirán muchos trabajadores del conocimiento en el sector NBIC, y no parece que el actual sistema de educación en ciencia y tecnología sea capaz de generarlos.

Este segundo factor resulta decisivo para entender por qué, en lugar de intentar atraer a más jóvenes al campo NBIC, se optó por una propuesta mucho más radical: reformar por completo el sistema educativo norteamericano e introducir el campo NBIC desde la escuela primaria, modificando para ello los planes de estudio. Las expectativas del sector NBIC son tan grandes, y las inversiones a realizar por parte de las empresas tecnocientíficas tan voluminosas, que se requiere tener garantías de que luego habrá suficientes personas capacitadas para trabajar en el sector.

Pero hay un tercer factor a tener en cuenta, la interdisciplinariedad, que aquí se menciona explícitamente como un objetivo a conseguir en dicha reforma educativa, en la que habrá que combatir la actual estructura, basada en le especialización disciplinaria, sustituyéndola por institucionesy programas educativosque promuevan decididamente la interdisciplinariedad. Bien entendido que esa promoción de la interdisciplinariedad ha de ser selectiva, lo cual supone un cuarto detalle a tener en cuenta a la hora de analizar el tratamiento de la interdisciplinariedad que ofrece el programa norteamericano CTIHP: ninguna de las disciplinas que se mencionan como integrantes del complejo interdisciplinario NBIC pertenecen al ámbito de las ciencias sociales, de las humanidades o de las artes. El núcleo duro del programa educativo CTIH P está conformado claramente por unas cuantas ingenierías y "ciencias duras".

Reinterpretadas las propuestas anteriores en nuestro propio marco conceptual, expresaremos el razonamiento subyacente del modo siguiente: expresaremos así: puesto que las empresas tecnocientíficas públicas y privadas que promueven la convergencia NBIC preveen a medio plazo una escasez de trabajadores capacitados en dicho sector de la economía del 
conocimiento, se exige a las autoridades educativas una macro-acción radical con el fin de resolver ese grave problema. El problema de fondo es la escasez de futuros trabajadores del conocimiento en el sector N BIC. Dichos trabajadores han de haber sido educados en la interdisciplinariedad, cosa que el actual sistema educativo no hace. Por tanto, la interdisciplinariedad es un objetivo a lograr, pero no por razones que tengan que ver con el conocimiento científico, sino con las necesidades de "mano de obra" de las empresas del sector, que ciertamente son poderosas e influyentes. En suma, el fomento de la interdisciplinariedad es un medio para el logro de determinados objetivos estratégicos, tanto por parte de las agencias públicas como de las empresas privadas del sector NBIC.

La interpretación que muy sucintamente acabamos de presentar configura la tesis final de este artículo. Su validez puede verse apoyada por numerosos pasajes de los dos informes de la NSF. M encionaremos dos más, procedentes del resumen ejecutivo del segundo informe. Ambos revelan otros matices importantes del enfoque que subyace a la gran reforma educativa propuesta:

Education in nanoscience and nanotechnology requires special laboratory facilities that can be quite expensive. Given the cost of creating and sustaining such facilities, their incorporation into nanotechnology workforce development presents a considerable challenge. Under the present education system, many engineering schools, letalone the two-year-degree colleges, cannotoffer students any exposure to the practice of nanofabrication. (RO CO ; BAIN BRIDGE, 2001, Societal Implications, p. 13)

$A$ related educational challenge is the very small number of social scientists who have the technical background and research orientation that would allow them to conduct competent research on the societal implications of nanotechnology. At the university level, liberal arts 
education gives far too low a priority to scientific literacy. Social science professional societies, universities, and government agencies will have to make a long-term commitment to attract talented young social scientists to this area of research and to encourage them to gain the necessary professional skills and awareness of nanotechnology. This will require research on the societal implications of nanotechnology at a consistent and high enough level to establish this as a viable field of social science research. (ROCO; BAINBRIDGE, 2001, Societal Implications, p. 14)

La primera cita señala un problema muy importante: cómo sería la enseñanza práctica de las NBIC en las universidadesy cómo podrían costearse y mantenerse los laboratorios docentes correspondientes, que serían muy caros. Por nuestra parte añadiríamos que también habría que analizar los riesgosque podrían suponer la instalación de ese tipo de laboratorios en los campus universitarios, y qué precauciones deberían tomar los estudiantesa la hora de manipular las nanopartículas y los nanotubos, en algunas de cuyas variantes se han constatado riesgos significativos (Royal Society and Royal Engineering Report, 2004). El informe europeo CTEKS se manifiesta mucho más sensible a la toma en consideración e investigación de los riesgos N BIC que el norteamericano, para el que sólo parecen existir las oportunidades.

La segunda cita puede resultar incluso más ilustrativa para el tema de este artículo, porque muestra que la interdisciplinariedad que se promueve en el informe CTIHP no se refiere a las ciencias sociales y humanas, y mucho menos a las artes. Se trata, como ya dijimos, de una interdisciplinariedad selectiva. Eso sí, se admite que algunos científicos sociales, siempre que tuvieran la adecuada formación NBIC, podrían investigar las implicaciones sociales de la convergencia NBIC que, conforme al informe CTIHP, habrá de producirse inexorablemente en las próximas décadas. Pero ello no implica que dichos científicos sociales van a formar parte de los grupos de 
investigación que generen nuevo conocimiento en el ámbito NBIC. Por tanto, no se considera necesario que ese tipo de habilidades formen parte del núcleo educativo NBIC, a lo sumo serían destrezas complementarias.

En resumen, podemos concluir que la interdisciplinariedad que se promueve en el programa norteamericano CTIHP es, en primer lugar, instrumental. Así como la especialización de las disciplinas fue adecuada en la época industrial, está dejando de serlo para el desarrollo de las economías y empresas de la información y el conocimiento, razón por la cual hay que favorecer la interdisciplinariedad en los diversos niveles educativos. En segundo lugar, dicha interdisciplinariedad es muy selectiva, porque sólo afecta a algunas disciplinas científicas e ingenieriles. Las ciencias sociales, las humanidades y las artes habrían de hacer suya la interdisciplinariedad NBIC, en la medida en que pretendan tener ámbitos de investigación vivos en dicho dominio. En tercer lugar, el informe CTIHP manifiesta fuertes tendencias reduccionistas y unificadoras, que no impulsan la interdisciplinariedad, sino la reducción de la mayoría de las disciplinas a unas pocas (NBIC), incluyendo los aspectos conceptuales y metodológicos. Esta tercera conclusión resulta más difícil de argumentar, porque el informe CTIHP nunca justifica la necesidad ni la conveniencia de la unifying science que promueve, pero hay numerosos detalles y afirmaciones que apoyan una interpretación así. Por estas tres razones pensamos que, aunque en dicho informe se habla una y otra vez a favor de la interdisciplinariedad, dichas afirmaciones resultan muy cuestionables, dado el transfondo selectivo, cientifista y reduccionista que se manifiesta en los pasajes del informe en los que se abordan cuestiones educativas.

Mantenemos, por tanto, la hipótesis general: la tecnociencia promueve la interprofesionalidad, más que la interdisciplinariedad, y lo hace por varios mecanismos. La misma elaboración de los dos informes que estamos comentando, en los que los debates parecieron ser escasos y los consensos 
abundantes, muestra que el "contrato social de la ciencia" del que habló Bush sigue vigente en el caso de la convergencia tecnológica nano-bio-info-cogno. Aunque en principio pudiera parecer que dicha convergencia promueve la interdisciplinariedad, el concepto mismo de interdisciplinariedad ha cambiado radicalmente, al igual que la propia noción de ciencia. En concreto, la gran transformación de la estructura académica tradicional de la ciencia promovida por losinformes de la N SF utiliza la interdisciplinariedad como un instrumento útil para el logro de los objetivos de los programas Converging Technologies. El fondo de la cuestión no está en la interdisciplinariedad, sino en la organización del trabajo en las empresas del conocimiento del sector NBIC.

\title{
Interdisciplinarity and the nano-bio-info-cogno technoscientific convergence
}

\begin{abstract}
The emergence of technoscience in the twentieth century changed scientific practice and the concept of interdisciplinarity. This article examines an important example of contemporary technoscience, which is the National Science Foundation (NSF) report Converging Technologies for improving Human Performance (CTIHP), and its support for a new kind of interdisciplinarity, the nano-bio-info-cogno (NBIC) technologies. The analysis of this report shows that the NSF concept of interdisciplinarity is instrumental, selective, and apparently reductionist, in detriment of the social sciences, artsand humanities. In the thesis, it is suggested that the concept of interprofessionality is more suitable for technoscience than that of interdisciplinarity. Keywords: Interdisciplinariety. Converging technologies.Nano-bio-info-cogno technoscience. Interprofesionality.
\end{abstract}

\section{Referencias}

BUSH, V., Science: the Endless Frontier. Washington: United States Government Printing, 1945. 
CASTELLS, M . et alia, La transición a la sociedad red. Barcelona: Ariel,2007. DRU CKER, P. Knowledge work and knowledge society. JFK School of Government, Harvard University, 1994

DRU CKER, P. The Discipline of Innovation. Claremont:0 Drucker Foundation News,1998.

EC. Nanotechnologies: A Preliminary Risk Analysis. Bruselas: UE, Report,2004. ECHEVERRÍA, J. Ciencia y Valores. Barcelona: Destino, 2002.

ECHEVERRÍA, J. La revolución tecnocientífica. M adrid: FCE, 2003.

FEYNM AN, R. P. There's Plenty of Room at the Bottom. en H. D. GILBERT (ed.), Miniaturization. New York: Reinhold Publ. Corp, 1961. p. 282-296

GIBBO NS, M.; C. Limoges; H. Nowotny; S. Schwartzman; P. Scott; M. Trow. The new production of knowledge. The dynamics of science and research in contemporary societies. Sage Publications, 1994.

LATO UR, B. Ciencia en Acción. Barcelona: Labor, 1992.

LUNDVALL, B.A. National systems of Innovation: Towards a theory of interactive learning. London: Pinter,1992.

MERTO N, R. K. La Sociología de la Ciencia. Madrid: Alianza, 2 vols, 1977.

NORDMANN, A. (coord.). Converging Technologies: Shaping the Future of the European Societies. Bruselas: UE. 2004.

O LIVÉ, L. La ciencia y la tecnología en la sociedad del conocimiento. México: FCE, 2007.

PICKERING, A. (ed.). Science as Practice and Culture. Chicago: University of Chicago, 1992.

PICKERING, A. The Mangle of Practice. Chicago: Univ. of Chicago Press, 1995.

RO CO, M. S. ; W. S. Bainbridge (eds.). Converging Technologies for Improving Human Performance. Virginia: NSF, 2001.

RO CO , M. S. ; W. S. Bainbridge, (eds.). Societal Implications of Nanoscience and Nanotechnology.Virginia: NSF, 2001.

The Royal Society ; The Royal Academy of Engineering. Nanoscience and nanotechnologies: opportunities and uncertainties. London: British Government, 2004. SO LLA, Price; D. Della. Hacia una ciencia de la ciencia. Barcelona: Ariel, 1973. ZIMAN, J. Real Science. Cambridge: Cambridge Univ. Press, 2000.

Recebido: $11 / 01 / 2008$ Aceite final: 14/01/2009 